\title{
INFLUENCIA DE LA EDUCACIÓN FINANCIERA CORPORATIVA SOBRE LA TOMA DE DECISIONES EN EMPRESARIOS PROCESADORES DE ACEITUNA Y DERIVADOS REGIÓN TACNA, 2016
}

\author{
INFLUENCE OF CORPORATE FINANCIAL EDUCATION ON DECISION-MAKING IN OLIVE \\ PROCESSING COMPANIES AND DERIVATIVES TACNA REGION, 2016
}

\section{Edgard Enrique Wong Copaja²}

\section{RESUMEN}

Objetivo: La presente tesis tuvo por objetivo determinar la influencia de la educación financiera corporativa sobre la toma de decisiones en empresarios procesadores de aceituna y derivados Región Tacna, 2016.

Metodología: El tipo de investigación es pura, porque está orientada a lograr un nuevo conocimiento en el campo de la finanza. La presente investigación responde a un diseño de investigación descriptiva causal explicativa. La población está conformada por todos los gerentes de empresas procesadoras de Aceituna y Derivados del Sector Agro Industrial de la región de Tacna. La muestra es censal y toma a los 15 gerentes de empresas procesadoras de Aceituna y Derivados del Sector Agro Industrial de la Región de Tacna que se encuentran Registradas en la Cámara de Comercio de Tacna. La técnica para la Recolección de los datos para la presente investigación fue la encuesta. El instrumento que se aplicó fue el cuestionario con la escala de Likert. Para el análisis estadístico se aplicó el Chi-Cuadrado, y otras medidas de tendencia central.

Resultados: Los resultados encontrados en la investigación fueron que el $53.3 \%$ de los empresarios procesadores de aceituna y derivados no cuentan con una educación financiera corporativa y de ellos el $60 \%$ de los empresarios siguen tomando decisiones empíricas.

Conclusión: Se comprueba en la presente tesis, que los empresarios procesadores de aceituna y derivados de la región de Tacna, no cuentan con una educación financiera corporativa adecuada y en consecuencia no están capacitados para realizar presupuestos, administrar deudas, invertir y ahorrar.
Palabras claves: Educación financiera corporativa, toma de decisiones, rentabilidad.

\section{ABASTRACT}

Objective: The purpose of this thesis was to determine the influence of corporate financial education on decision - making in olive processing entrepreneurs and derivatives Region Tacna, 2016.

Methodology: The type of research is pure, because it is oriented to achieve a new knowledge in the field of finance. The present research responds to an explanatory causal descriptive research design. The population is conformed by all the managers of processing companies of Olive and Derivatives of the Agro Industrial Sector of the region of Tacna. The sample is census and takes the 15 managers of processing companies of Olive and Derivatives of the Agro Industrial Sector of the Region of Tacna that are registered in the Chamber of Commerce of Tacna. The technique for the collection of the data for the present investigation was the survey. The instrument that was applied was the questionnaire with the Likert scale. Statistical analysis was applied ChiSquare, and other measures of central tendency.

Results: The results found in the research were that $53.3 \%$ of the olive and derivative processors do not have a corporate financial education and $60 \%$ of the entrepreneurs still make empirical decisions.

Conclusion: As a conclusion of this thesis, the processors of olives and derivatives from the region of Tacna do not have adequate corporate financial education and this is a consequence of not being able to make budgets, debt management, investment, Saving.

Key words: Corporate financial education, decision making, profitability 


\section{INTRODUCCION}

Rodriguez, (2012), hace incapie que más del 58\% de los empresarios estan tomando mejores decisiones en base a una educacion financiera adecuada dando valor a su empresa. Curbelo (2012), determino que existe un crecimiento del $51 \%$ de concientización mundial sobre la necesidad de promover cambios positivos en los niveles de educación financiera de los individuos y las empresas. Esa conciencia es consecuencia de varios factores, entre los que se incluyen los retos económicos y la evidencia de bajos niveles de educación financiera. Rijardo (2012), señala que la educación financiera contribuye a reducir la mala toma de decisiones contra la crisis económicas. Según la Superintendencia de Banca del Perú (2012), los beneficios de la educación financiera también pueden extenderse a la economía en general. Por la cual la educación financiera puede promover las competencias necesarias para tomar decisiones informadas y apropiadas, así como proporcionar herramientas para que las personas tengan la capacidad de defender sus derechos como consumidores financieros. Hoy en día la economía nacional goza de altos índices de crecimiento económico que permiten que los empresarios puedan tener mayores ingresos económicos. (Cepal, 2016). A pesar de ello, los empresarios peruanos no poseen cultura financiera, lo cual se entiende en la forma como gestionan sus negocios. Muchos de estos enfocan más sus gestiones en la parte financiera haciendo de esto la parte más importante, dejando a un lado otras actividades que podrían contribuir a los logros de las mismas. En la ciudad de Tacna tal realidad no es ajena ya que el $82 \%$ de los empresarios no toman decisiones acertadas y solo se basan en opiniones empíricas (Albarracin, 2015).

Se percibe que los empresarios exportadores de aceituna y derivados no toman decisiones adecuadas, esto se refleja en su inconformidad con el desempeño general de sus empresas porque presentan perdidas, no generan suficiente valor, o muchas veces al implementar nuevas formas de administrar sus negocios, olvidan algunas cosas o preguntas, que deben estar claras para marcar el rumbo de la organización.

Según (Buffett W. , 2013) en su libro Aprende a invertir coloca cuatro puntos sobre la importancia de la educación financiera.

- $\quad$ Ayuda a las personas a utilizar productos y servicios financieros que mejoren su calidad de vida bajo condiciones de certeza.

- $\quad$ Ayuda a la persona a cuidar y hacer rendir su dinero, y a utilizar adecuada y responsablemente los productos y servicios financieros.

- $\quad$ Ayuda a la familia a obtener mayor oportunidad de generar los recursos necesarios para su estabilidad y desarrollo.

- Genera empresarios más informados y exigentes, lo que promueve la competitividad entre las empresas, y por ello, un beneficio directo a los mercados financieros, traduciéndose en mayor desarrollo para el país.

Respecto de los beneficios de la Educación Financiera Corporativa se tiene lo siguiente:

- $\quad$ Mejor calidad de vida al tener la capacidad de generar riqueza.

- Selección inteligente de los distintos financiamientos (crédito), poniendo atención en la tasa de interés, las comisiones, así como los beneficios de pagar a tiempo.

- $\quad$ Buena salud al tener menos estrés derivado de la preocupación de qué se va a hacer para cubrir los gastos del mes o las deudas.

- $\quad$ Realización de metas patrimoniales: casa, auto, negocio, retiro cómodo, educación de los hijos, etcétera.

- $\quad$ Manejo óptimo del crédito en la construcción del patrimonio.

- $\quad$ Finanzas estables y preparadas para enfrentar cualquier contingencia (crisis), porque se cuenta con un fondo de emergencia y diversificación de ingresos. 
- Mejor selección de instrumentos financieros para que nuestras inversiones obtengan los mejores rendimientos con menor riesgo. (Eker, 2010).

En cuanto a la variable toma de decisiones, según Houch (2001), sostiene que la toma de decisiones es un proceso cognitivo que resulta en la selección de un curso de acción entre varias posibilidades alternativas. Cada proceso de toma de decisiones produce una decisión final que puede o no puede incitar acción. La toma de decisiones es el proceso de identificación y elección de alternativas basadas en los valores y preferencias del decisor.

Por otro lado Gunteher (2007), sostiene que la Toma de decisiones dinámicas, tiene lugar en un entorno que cambia con el tiempo, ya sea debido a las acciones anteriores de la toma de decisiones o debido a acontecimientos que están fuera del control de la toma de decisiones. En este sentido, las decisiones dinámicas, a diferencia de las decisiones simples y convencionales de una sola vez, suelen ser más complejas y se producen en tiempo real e implican observar el grado en que las personas son capaces de utilizar su experiencia para controlar un determinado sistema complejo, incluyendo los tipos de experiencia que llevan a mejores decisiones en el tiempo.

Según Saaty (2002), La toma de decisiones es el proceso mediante el cual se realiza una elección entre las opciones o formas para resolver diferentes situaciones de la vida en diferentes contextos: a nivel laboral, familiar, personal, sentimental o empresarial. Por su parte, Stoner (2003), Define la toma de decisiones como "el proceso para identificar y solucionar un curso de acción para resolver un problema específico". Según Fremont E.Kast, la toma de decisiones es fundamental para el organismo y la conducta de la organización, la toma de decisiones suministra los medios para el control y permite la coherencia en los sistemas.

\section{MATERIALES Y MÉTODOS}

La presente investigación es un tipo de investigación pura, porque está orientada a lograr un nuevo conocimiento en el campo de la finanza. La presente investigación por su naturaleza de las variables responde a un diseño de investigación descriptiva causal explicativa. El espacio de intervención de la investigación es regional. Los resultados de la investigación tendrán vigencia y validez regional, provincial y nacional ya que ayudara a los empresarios a tomar mejores decisiones para rentabilizar su empresa, a crear valor. La población está conformada por todos los gerentes de empresas procesadoras de Aceituna y Derivados del Sector Agro Industrial de la región de Tacna. La muestra es censal y toma a los 15 gerentes de empresas procesadoras de Aceituna y Derivados del Sector Agro Industrial de la Región de Tacna que se encuentran Registradas en la Cámara de Comercio de Tacna. La técnica para la Recolección de los datos para la presente investigación fue la encuesta. La encuesta consta de 7 preguntas relacionadas a los datos generales, 23 preguntas relacionadas a la educación financiera corporativa y 12 preguntas relacionadas a la toma de decisiones. El instrumento que se aplicó fue el cuestionario con la escala de Likert. En la tesis se ha utilizado el estadístico Chi-Cuadrado, y otras medidas de tendencia central y los parámetros de la población.

\section{RESULTADOS}

\section{EDUCACIÓN FINANCIERA CORPORATIVA}

Con la finalidad de conocer al detalle las características de cada una de las variables en estudio, se mostrara los cuatro indicadores de la educación financiera corporativa. 
Tabla 1: Nivel de Inversión

\begin{tabular}{lcc}
\hline Categoría & Recuento & $\%$ \\
\hline Bajo & 4 & $26.7 \%$ \\
Medio & 6 & $40.0 \%$ \\
Alto & 5 & $33.3 \%$ \\
Total & 15 & $100.0 \%$ \\
\hline
\end{tabular}

Fuente: Encuesta dirigida a los empresarios procesadores de aceituna y derivados de la región de Tacna, 2016

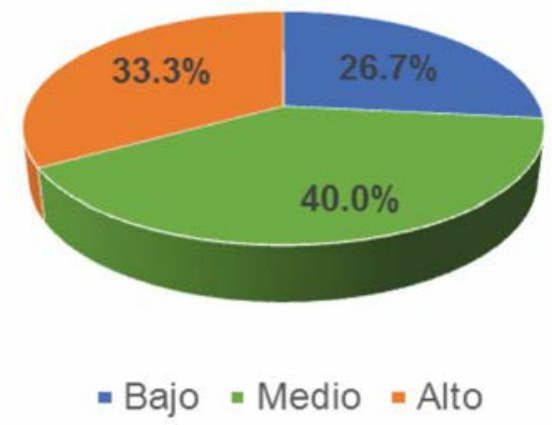

Figura 1: Nivel de Inversión

Fuente: Elaboración Propia, Tabla 1

\section{INTERPRETACIÓN}

Según la tabla 1 y figura 1 , se muestran el Nivel de Inversión que tienen los empresarios procesadores de aceituna y derivados de la región de Tacna de lo cual el $40 \%$ de los empresarios muestran un nivel medio de inversión, a si mimo un 33.3\% muestra un alto Nivel de inversión lo cual quiere decir que se preocupan por invertir en la empresa, pero por otro lado un $26.7 \%$ tiene un Nivel Bajo lo cual quiere decir que no Invierte en su empresa.

Tabla 2: Nivel de Inversión

\begin{tabular}{|c|c|c|c|}
\hline & Media & Desviación estándar & Categoría \\
\hline $\begin{array}{l}\text { Las inversiones que } \\
\text { realizó tienen el } \\
\text { rendimiento esperado }\end{array}$ & 1.20 & 0.414 & Siempre \\
\hline $\begin{array}{l}\text { Invierto mi capital en } \\
\text { negocios con } \\
\text { rentabilidad por } \\
\text { debajo del porcentaje } \\
\text { aceptado }\end{array}$ & 1.67 & 0.488 & Nunca \\
\hline $\begin{array}{l}\text { Considero que la } \\
\text { inversión que realizo } \\
\text { tendrá un impacto } \\
\text { positivo en el } \\
\text { rendimiento esperado }\end{array}$ & 1.20 & 0.414 & Siempre \\
\hline $\begin{array}{l}\text { En que invirtio } \\
\text { Porque considera que }\end{array}$ & 2.53 & 1.407 & Maquinaria \\
\hline $\begin{array}{l}\text { la inversión que } \\
\text { realiza tendrá un } \\
\text { rendimiento positivo }\end{array}$ & 5.07 & 3.058 & Realizo Planeamiento \\
\hline
\end{tabular}

Fuente: Elaboración propia, Encuesta dirigida a los empresarios procesadores de aceituna y derivados de la región de Tacna, 2016 


\section{INTERPRETACIÓN}

Según la tabla 2 se muestra los criterios de inversión que los empresarios siempre tienen en cuenta cuando invierten, consideran el rendimiento esperado que desean, a su vez los empresarios nunca invierten su capital en negocios por debajo del rendimiento aceptado, por otro lado los empresarios siempre consideran que las inversiones que realizan tienen un impacto positivo sobre la rentabilidad, a su vez los empresarios siempre invierten en maquinaria, por otro lado siempre consideran que la inversión que realizan tendrá un rendimiento positivo sobre sus activos porque realizar un planeamiento de sus inversiones.

Tabla 3 : Nivel de Ahorro

\begin{tabular}{lcc}
\hline Categoría & Recuento & $\%$ \\
\hline Bajo & 7 & $46.7 \%$ \\
Medio & 2 & $13.3 \%$ \\
Alto & 6 & $40.0 \%$ \\
Total & 15 & $100.0 \%$ \\
\hline
\end{tabular}

Fuente: Encuesta dirigida a los empresarios procesadores de aceituna y derivados de la región de Tacna, 2016

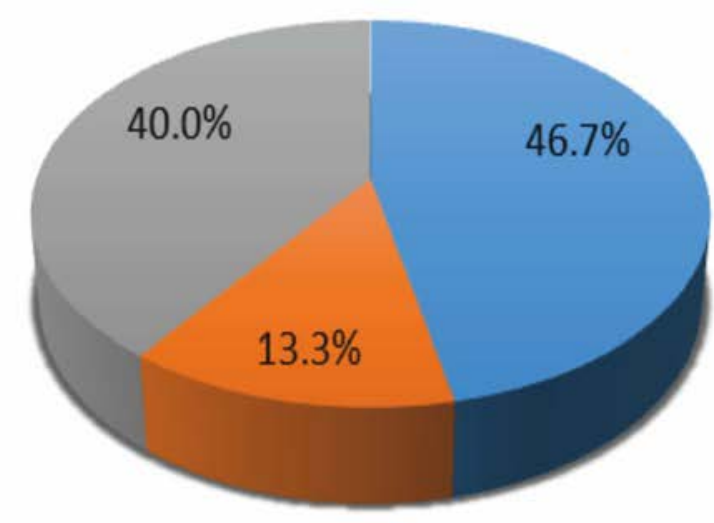

\section{= Bajo = Medio = Alto}

Figura 2. Nivel de Ahorro

Fuente: Elaboración Propia, Tabla 3

\section{INTERPRETACIÓN}

Según la tabla 3, se muestran el Nivel de ahorro que tienen los empresarios procesadores de aceituna y derivados de la región de Tacna, en el cual se observa que el $46.7 \%$ de los empresarios tienen un bajo nivel de ahorro, por otra parte un $40 \%$ de los empresarios tienen un alto nivel de ahorro, a su vez un $13.3 \%$ tienen un nivel medio de ahorro.

Por lo cual se puede asegurar que los empresarios procesadores de aceituna y derivados de la región de Tacna, se preocupan por reservar activos para respaldar algunos inconvenientes o percances que sucedan en la empresa. 
Tabla 4. Items de Nivel de Ahorro

\begin{tabular}{|c|c|c|c|}
\hline & Media & Desviación estándar & Categoría \\
\hline $\begin{array}{l}\text { Reservó un } \\
\text { porcentaje de las } \\
\text { utilidades para una } \\
\text { futura inversión o } \\
\text { actividad inesperada }\end{array}$ & 1.33 & .488 & Siempre \\
\hline $\begin{array}{l}\text { En qué actividades } \\
\text { invierto }\end{array}$ & 2.47 & 1.407 & Materia Prima \\
\hline $\begin{array}{l}\text { Cuanto fue el } \\
\text { porcentaje que } \\
\text { destino en la } \\
\text { Inversión }\end{array}$ & 3.53 & 2.264 & $30 \%$ \\
\hline $\begin{array}{l}\text { Utilizo fondos de } \\
\text { inversión dentro de } \\
\text { mi empresa }\end{array}$ & 1.53 & .516 & A veces \\
\hline $\begin{array}{l}\text { En que utiliza los } \\
\text { fondos de inversión } \\
\text { Utilizo medios }\end{array}$ & 1.93 & 1.280 & $\begin{array}{l}\text { Compra de } \\
\text { Maquinaria }\end{array}$ \\
\hline $\begin{array}{l}\text { electrónicos para } \\
\text { realizar pagos }\end{array}$ & 1.53 & .516 & A veces \\
\hline $\begin{array}{l}\text { En qué ocasión } \\
\text { utilizo el internet } \\
\text { para hacer sus } \\
\text { pagos }\end{array}$ & 2.00 & 1.254 & $\begin{array}{l}\text { Compra de } \\
\text { Maquinaria }\end{array}$ \\
\hline
\end{tabular}

Fuente: Encuesta dirigida a los empresarios procesadores de aceituna y derivados de la región de Tacna, 2016

\section{INTERPRETACIÓN}

Según la tabla 4, se muestra sobre los ítems de nivel de ahorro, que los empresarios siempre reservan un porcentaje de las utilidades para una futura inversión o actividad inesperada, a su vez los empresarios invierten en materia prima, y utilizan el $30 \%$ del ahorro para inversiones como la compra de maquinaria y a veces utilizan medios electrónicos para comprar maquinarias en el extranjero.

\section{Tabla 5. Nivel de Administración de la Deuda}

\begin{tabular}{lcc}
\hline Categoría & Recuento & $\%$ \\
\hline Bajo & 10 & $66.7 \%$ \\
Medio & 5 & $33.3 \%$ \\
Alto & 0 & $0.0 \%$ \\
Total & 15 & $100.0 \%$ \\
\hline
\end{tabular}

Fuente: Encuesta dirigida a los empresarios procesadores de aceituna y derivados de la región de Tacna, 2016

\section{INTERPRETACIÓN}

Según la tabla 5 se observa que el $66.7 \%$ de los empresarios procesadores de aceituna y derivados de la región de Tacna, tienen un bajo nivel de administración de la deuda, pero a sí mismo un $33.3 \%$ de los empresarios tienen un nivel medio en administración de la deuda.

Por lo tanto, se concluye que los empresarios procesadores de aceituna y derivados de la región de Tacna, no se están preocupando por llevar a la empresa por la buena y correcta organización por lo que no pueden mejorar su Rentabilidad. 
Tabla 6. Nivel de Presupuesto

\begin{tabular}{lrr}
\hline \multicolumn{1}{c}{ Categoría } & Recuento & \multicolumn{1}{c}{$\%$} \\
\hline Bajo & 7 & $46.7 \%$ \\
Medio & 0 & $0.0 \%$ \\
Alto & 8 & $53.3 \%$ \\
Total & 15 & $100.0 \%$ \\
\hline
\end{tabular}

Fuente: Encuesta dirigida a los empresarios procesadores de aceituna y derivados de la región de Tacna, 2016

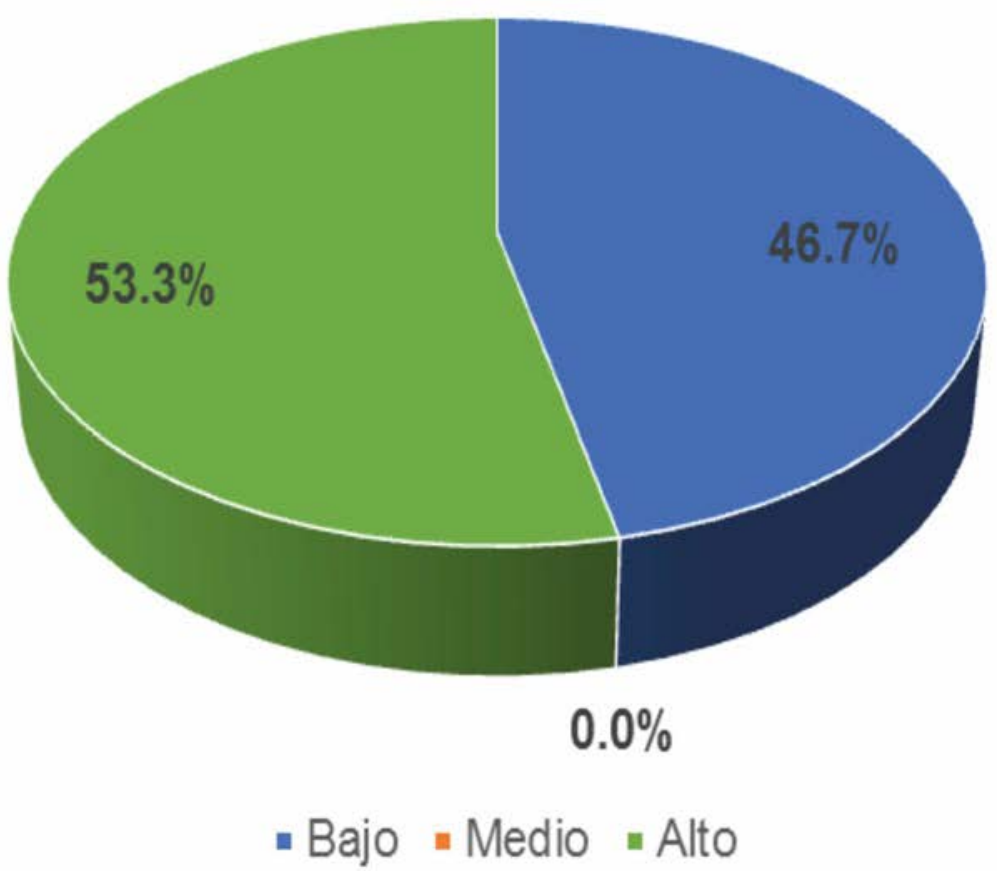

Figura 3.Nivel de Presupuesto

Fuente: Elaboración Propia, Tabla 6

\section{INTERPRETACIÓN}

La tabla 6 y figura 3 muestran el nivel de presupuesto que tienen los empresarios procesadores de aceituna y derivados de la región de Tacna. El 53.3\% de los empresarios siguen un orden y una organización para el presupuesto anual de su empresa pero así mismo un $46.7 \%$ muestra que los empresarios no acostumbran a realizar una organización del presupuesto anual. Por lo cual se puede asegurar que los empresarios procesadores de aceituna y derivados de la región de Tacna, se organizan para realizar el presupuesto anual de su empresa para no tener ningún inconveniente en el transcurso del tiempo. 
Tabla 7. Items de Nivel de Presupuesto

\begin{tabular}{|c|c|c|c|}
\hline & Media & Desviación estándar & Categoría \\
\hline $\begin{array}{l}\text { Realizo una previa } \\
\text { planeación del } \\
\text { presupuesto que se va } \\
\text { a usar en el año }\end{array}$ & 1.47 & .516 & A veces \\
\hline $\begin{array}{l}\text { Organizo actividades } \\
\text { para la elaboración del } \\
\text { presupuesto anual }\end{array}$ & 1.53 & .516 & A veces \\
\hline $\begin{array}{l}\text { Que actividades fueron } \\
\text { fundamentales para } \\
\text { elaborar su } \\
\text { presupuesto }\end{array}$ & 2.07 & 1.486 & $\begin{array}{c}\text { Ingresos y Egresos } \\
\text { del año pasado }\end{array}$ \\
\hline $\begin{array}{l}\text { Realizo una } \\
\text { coordinación previa } \\
\text { con sus gerentes de } \\
\text { área para el armado } \\
\text { de cronograma de } \\
\text { actividades anuales }\end{array}$ & 1.47 & .516 & Casi Siempre \\
\hline $\begin{array}{l}\text { Considera que existe } \\
\text { una relación entre el } \\
\text { progreso de la } \\
\text { empresa debido a la } \\
\text { dirección y } \\
\text { calendarización del } \\
\text { presupuesto anual }\end{array}$ & 1.47 & .516 & Casi Siempre \\
\hline $\begin{array}{l}\text { Como gerente general } \\
\text { organiza juntas para } \\
\text { ver el rendimiento del } \\
\text { presupuesto anual }\end{array}$ & 1.47 & .516 & Casi Siempre \\
\hline
\end{tabular}

Fuente: Encuesta dirigida a los empresarios procesadores de aceituna y derivados de la región de Tacna, 2016

\section{INTERPRETACIÓN}

Según la tabla 7, sobre los ítems de nivel de presupuesto, se muestra que, el empresario a veces realiza una planeación del presupuesto que se va a usar en el año, y que a veces organiza actividades para la elaboración del presupuesto, por otro lado las actividades que fueron fundamentales para la elaboración del presupuesto fueron los ingresos y egresos del año pasado, y que casi siempre realiza una coordinación previa con sus gerentes de área para el armado de cronogramas de actividades anuales, y considera que existe una relación entre el progreso de la empresa debido a su dirección y calendarización, por otro lado casi siempre como gerente general organiza juntas para ver el rendimiento del presupuesto anual.

Tabla 8. Resumen del Nivel de Educación Financiera Corporativa

\begin{tabular}{lrr}
\hline Categoría & Recuento & \multicolumn{1}{c}{$\%$} \\
\hline Bajo & 5 & $53.3 \%$ \\
Medio & 4 & $20.7 \%$ \\
Alto & 6 & $26.7 .0 \%$ \\
Total & 15 & $100.0 \%$ \\
\hline
\end{tabular}

Fuente: Encuesta dirigida a los empresarios procesadores de aceituna y derivados de la región de Tacna, 2016 


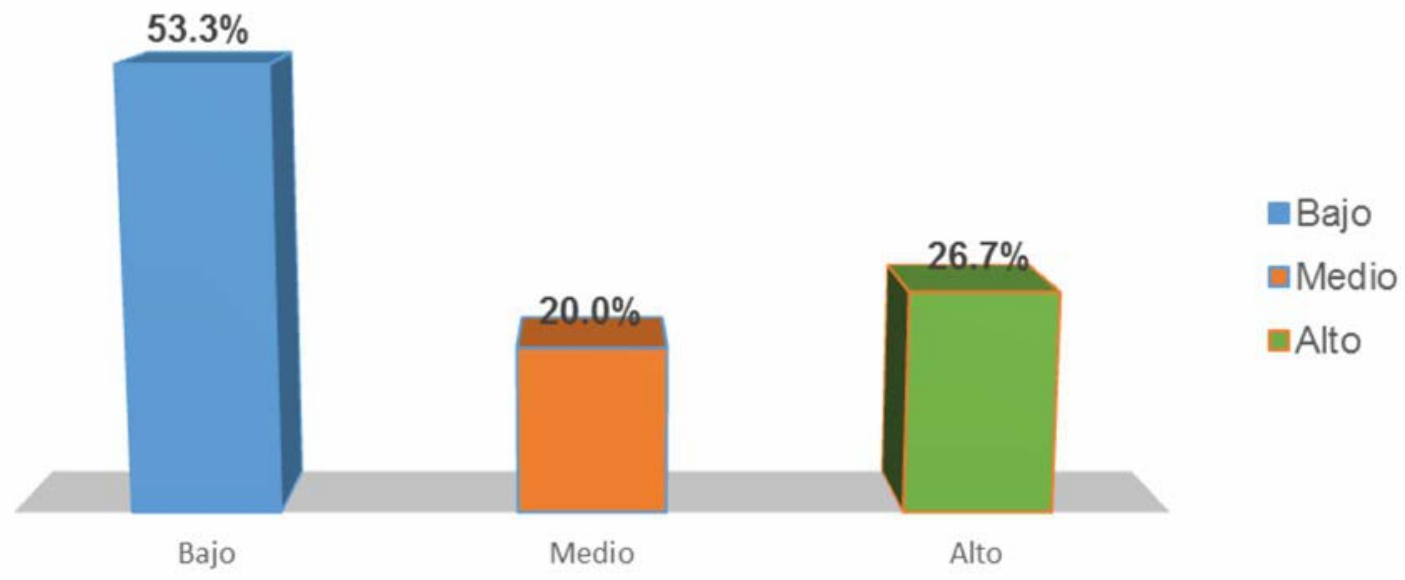

Figura 4. Resumen del Nivel de Educación Financiera Corporativa

Fuente: Elaboración Propia, Tabla 8

\section{INTERPRETACIÓN}

La tabla 8 y la figura 4, muestran los porcentajes sobre el Nivel de Educación Financiera Corporativa de los empresarios procesadores de aceituna y derivados de la región de Tacna. El $53.3 \%$ de los empresarios tienen un nivel de Educación Financiera Corporativa baja, así mismo un $26.7 \%$ de los empresarios tienen un nivel de educación financiera alta, a su vez un $20 \%$ muestra una educación financiera Corporativa Media.

Por lo cual se puede asegurar que los empresarios procesadores de aceituna y derivados de la región de Tacna tienen una educación financiera corporativa baja, y que es producto de no tener un buen nivel de ahorro, presupuesto, administración de la deuda y tampoco un nivel de inversión.

\section{TOMA DE DECISIONES}

Con la finalidad de conocer al detalle las características de la variable toma de decisiones, se mostrara el comportamiento de sus tres indicadores.

Tabla 9. Nivel de Análisis de Documentos

\begin{tabular}{lcc}
\hline Categoría & Recuento & $\%$ \\
\hline Bajo & 9 & $60.0 \%$ \\
Medio & 4 & $26.7 \%$ \\
Alto & 2 & $13.3 \%$ \\
Total & 15 & $100.0 \%$ \\
\hline
\end{tabular}

Fuente: Encuesta dirigida a los empresarios procesadores de aceituna y derivados de la región de Tacna, 2016

\section{INTERPRETACIÓN}

La tabla 9, muestran el nivel de análisis de documentos que tienen los empresarios procesadores de aceituna y derivados de la región de Tacna. Se observa que el $60 \%$ tienen un bajo nivel de análisis de documentos, el $26.7 \%$ tienen un nivel medio de análisis de documentos, y el $13.3 \%$ tiene un alto nivel de análisis de documentos. Por lo tanto, se puede concluir que los empresarios procesadores de aceituna y derivados de la región de Tacna, tienen un bajo nivel en utilizar el análisis de documentos para la toma de decisiones. 
Tabla 10. Nivel de Selección basado en criterios, tecnología e implementación

\begin{tabular}{lcc}
\hline Categoría & Recuento & $\%$ \\
\hline Bajo & 11 & $73.3 \%$ \\
Medio & 4 & $26.7 \%$ \\
Alto & 0 & $0.0 \%$ \\
Total & 15 & $100.0 \%$ \\
\hline
\end{tabular}

Fuente: Encuesta dirigida a los empresarios procesadores de aceituna y derivados de la región de Tacna, 2016

\section{INTERPRETACIÓN}

En la tabla 10 se muestran el nivel de selección basado en criterios, tecnología e implementación que tienen los empresarios procesadores de aceituna y derivados de la región de Tacna, Se observa que el $73.3 \%$ tienen un bajo nivel de selección, el $26.7 \%$ tiene un nivel medio de selección. Por lo descrito, se concluye que los empresarios consideran que los criterios que ellos están adoptando no les ayudan a poder mejorar e implementar tecnología de punta para su empresa.

Tabla 11. Nivel de implementación basado en gestión y capacitación

\begin{tabular}{lcc}
\hline Categoría & Recuento & $\%$ \\
\hline Bajo & 11 & $73.3 \%$ \\
Medio & 4 & $26.7 \%$ \\
Alto & 0 & $0.0 \%$ \\
Total & 15 & $100.0 \%$ \\
\hline
\end{tabular}

Fuente: Encuesta dirigida a los empresarios procesadores de aceituna y derivados de la región de Tacna, 2016

\section{INTERPRETACIÓN}

En la tabla 11 se muestran el nivel de implementación basado en gestión y capacitación, que tienen los empresarios procesadores de aceituna y derivados de la región de Tacna. El $73.3 \%$ tiene un bajo nivel de implementación por otro lado un 26.7\% tiene un Medio Nivel de implementación. De lo cual podemos asegurar que los empresarios no consideran que la capacitación constante ayuda a que la toma de decisiones será más efectiva, y que esto ayuda a mejor la gestión de la empresa.

\section{PRUEBA ESTADİSTICA}

Las pruebas estadísticas que se realizaron para la comprobación de la hipótesis general y de las hipótesis especifican se detallan a continuación:

Tabla 12. Nivel de Educación Financiera Corporativa

\begin{tabular}{lccc}
\hline & $\mathrm{N}$ & Media & $\begin{array}{c}\text { Desviación } \\
\text { estándar }\end{array}$ \\
\hline Nivel de & & & \\
$\begin{array}{l}\text { Educación } \\
\text { Financiera }\end{array}$ & 15 & 1.73 & .884 \\
Corporativa & & & \\
\hline Eorpation
\end{tabular}

Fuente: Elaboración propia, Encuesta dirigida a los empresarios procesadores de aceituna y derivados de la región de Tacna, 2016 


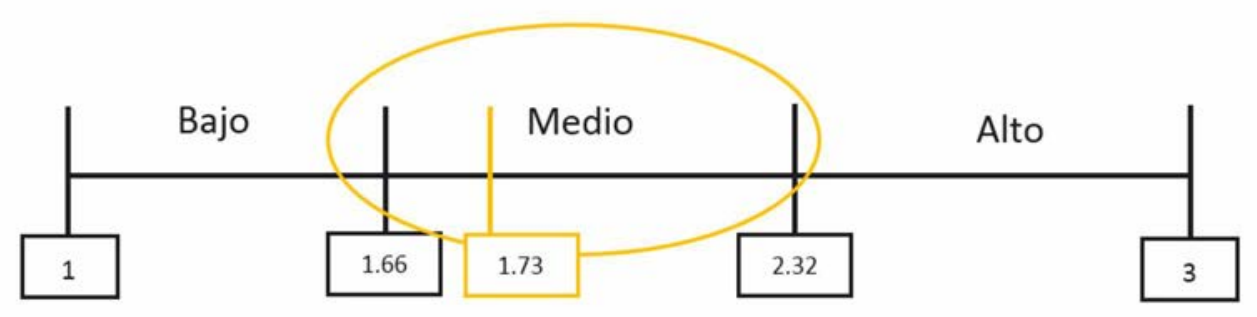

El valor del estadístico de la media de tendencia central es de 1.73 que cae en la zona del nivel medio, lo que significa que el nivel de educación financiera corporativa es media con el $95 \%$.

Tabla 13. Nivel de Toma De Decisiones

\begin{tabular}{lccc}
\hline & $\mathrm{N}$ & Media & $\begin{array}{c}\text { Desviación } \\
\text { estándar }\end{array}$ \\
\hline $\begin{array}{l}\text { Nivel de Toma } \\
\text { de Decisiones }\end{array}$ & 15 & 1.53 & .640 \\
\hline
\end{tabular}

Fuente: Encuesta dirigida a los empresarios procesadores de aceituna y derivados de la región de Tacna, 2016

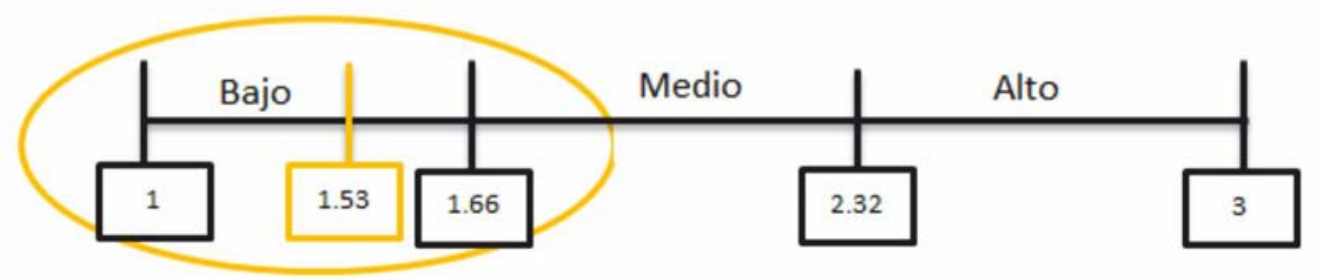

El valor del estadístico de la media de tendencia central es de 1.53 que cae en la zona de bajo nivel, lo que significa que el nivel de Toma de Decisiones es Bajo con el 95\%.

\section{CONCLUSIONES}

El $53.3 \%$ de los empresarios procesadores de aceituna y derivados de la región de Tacna no cuentan con educación financiera corporativa, caracterizándose que falta capacitarlos en realización de presupuesto, guía de ahorro, planificación de inversión, y un curso de administración de la deuda.

El $60 \%$ de los empresarios procesadores de aceituna y derivados de la región de Tacna cuentan con un bajo nivel de toma de decisiones, caracterizándolos a los empresarios con un Bajo análisis documentario lo cual no lo ayuda a poder tomar decisiones de implementación basado en gestión y tecnología.

Con la prueba de hipótesis Chi- Cuadrado se comprueba que la variable educación financiera corporativa tiene influencia sobre la variable toma de decisiones de los empresarios procesadores de Aceituna y derivados de la Región de Tacna, con un nivel de confianza del $95 \%$.

Los ítems de nivel de presupuesto, muestran que el $46.7 \%$ de los empresarios no sabe realizar un presupuesto y no cuentan con una guía para realizar una planeación de los costos e ingresos que van a obtener en el transcurso del año, esto origina sé que endeude y no cuente con una administración de la deuda correcta. 
Los ítems de nivel de administración de la deuda, muestra que el $66.7 \%$ de los empresarios no sabe cómo administrar la deuda, y que se encuentran solicitando créditos y microcréditos para seguir pagando deuda, y a su vez demuestra que el empresario sigue trabajando por dinero, en vez de que el dinero trabajo para él. Esto recae en que el empresario no cuenta con una guía de tasas de interés que te prestan los bancos y las amortizaciones de los intereses.

Los ítems de nivel de análisis de documentos, muestra que el $60 \%$ de los empresarios no toman decisiones mediante documentación y que contar con información actualizada no les facilita la toma de decisiones.

\section{REFERENCIAS BIBLIOGRÁFICAS}

Albarracin, R. G. (2015). Los cambios empresariales en la Región de Tacna. Tacna - Perú: Tacna.

Almaraz Rodríguez, I. (2007). Analisis de los Factores que Intervienen en la Toma de Decisiones de los Administradores dentro de las Organizaciones. Mexico: Universidad Autónoma de Querétaro. Recuperado el 3 de enero de 2016, de http://fca.uaq.mx/files/investigacion/doctorado/tesis/Almaraz_Rodriguez_Ignacio.pdf

Alpaca Salas, A. T. (2012). El Uso de Costos Relevantes para la Toma de Decisiones Gerenciales y su Influencia en la Utilidad de las empresas Industriales de la Ciudad de Tacna. Tacna: Universidad Nacional Jorge Basadre Grohmann.

Aroba Páez, J. (2013). Avances en la Toma de Decisiones en Proyectos de Desarrollo de Software. Perú: Universidad de Sevilla.

Becker Palmér, C. (2009). Los efectos de la cultura en Gestores de la toma de decisiones. Mexico y Alemania: Departamento de Ciencias de la Administracion de Negocios y Sociales Division del Marketing Industrial y el Comercio Electrónico.

Buffett, M. (2007). El Tao de Warren Buffett. Estados Unidos: Alienta.

Buffett, W. (2013). Aprende a invertir. USA: ALICE.

Carrascoza oliveriro, J. Q. (2009). Las finanzas personales como instrumento para el desarrollo de inversiones exitosas en el municipio de Joyabaj del departamento del Quiche. GuatemalaQuiche: Facultad de Ciencias Economicas de la Universidad de San Carlos de Guatemala.

Cepal. (10 de Enero de 2016). Crecimiento economico del Perú. (Cepal, Ed.) Lima, Lima, Perú.

Chiavenato, I. (2005). Administración de los Nuevos Tiempos. Brasil: Mc Graw Hill.

Comision Nacional de Defensa de los Usuarios de Servicios Financieros. (2010). Educacion Financiera en Mexico. Comision Nacional de Defensa de los Usuarios de Servicios Financieros, Mexico. Mexico: Comision Nacional de Defensa de los Usuarios de Servicios Financieros.

Comité Económico y Social Europeo. (2013). Union Europea. (U. d. Publicaciones, Ed.) Recuperado el 3 de enero de 2016, de Union Europea: http://www.eesc.europa.eu/resources/docs/qe-30-12-894-es.pdf

Curbelo, J. L. (2012). El Endeudamiento como Problematica Social Emergente. Chile: Grijalbo.

Denegri, D. M. (2009). PSICOLOGIA DEL ENDEUDAMIENTO. CHILE: P\&T.

Doval, I. (2009). La Ética en la Toma de Decisiones - La Consideración de los valores mediante la Acción Comunicativa. Argentina: Universidad de Buenos Aires. Recuperado el 3 de enero de 2016, de http://www.econ.uba.ar/www/servicios/biblioteca/bibliotecadigital/bd/tesis_doc/doval.pdf

Eker, T. (2010). Los secretos de la mente millonaria. USA : Aguilar.

Escott Mota, M. (2013). Estudio de la Educación Financiera del Personal Administrativo de la UAQ. Universidad Autónoma de Querétaro, Facultad de Contaduría y Administración. Mexico: Facultad de Contaduría y Administración. doi:http://ri.uaq.mx/bitstream/123456789/275/1/RI000027.pdf

F.Drucker, P. (2002). Las 6 Etapas para el proceso de la toma de decisiones. Austria: The Temptation to Do Good.

Flores Konja, A. (2004). Los Siete Pasos de Control de Calidad. Perú-Lima: Universidad Nacional Mayor de San Marcos. 
Franklin Marcelo, C. d. (2013). Los nuevos deudores del Peru. PERU: ARE.

Galdós Jiménez, W. G. (2014). Sesgos presentes en la toma de decisiones de los Gerentes y equipos gerenciales en lima-Perú. Lima: Universitat Politècnica de Catalunya. Departament d'Organització d'Empreses.

Garcia Gomez, Y. (2011). Caracteristicas e importancia de la educacion financiera para niños, jovenes y adultos de sectores populares de la ciudad de oaxaca de juarez. MexicoHuajuapan de leon: TCE.

Garcia, N. (2013). La Educacion Financiera en America Latina y El Caribe. Mexico: Corporacion Andina de Fomento.

Giráldez Betrón, J. I. (1999). Modelo de Toma de Decisiones y Aprendizaje en Sistemas MultiAgente. Madrid - España: Universidad Politecnica de Madrid. Recuperado el 3 de enero de 2016, de http://oa.upm.es/1255/1/10199908.pdf

Mejia, A. J. (2014). Inclusion de los beneficios que otorga la Educacion Financiera. Chile: Argos.

Moody, P. (1991). Toma de decisiones Gerenciales. Inglaterra: Mc Graw Hill.

Organizacion para la Cooperacion y el Desarrollo Economico. (2009). La Educacion Financiera Corporativa para los Empresarios. Mexico: OCDE.

Penfold, M. (2012). Situacion actual de la Educacion Mundial. Panama: Corporacion Andina de Fomento.

Puescas Chunga, P. (2013). El micro financiero (federación peruana de cajas municipales de ahorro y crédito del Perú). Perú - Lima: CMC.

Quiroa Morales, C. (2014). Toma de Decisiones y Productividad laboral. Universidad Rafael Landivar, Quetzaltenango. Guatemala: Universidad Rafael Landívar. doi:http://biblio3.url.edu.gt/Tesario/2014/05/43/Quiroa-Claudia.pdf

Rijardo, L. B. (2012). Toma de decisiones frente a las crisis economicas. USA: Works.

Rodríguez Cruz, Y. (2014). Modelo de uso de información para la Toma de Decisiones Estratégicas en Organizaciones de Informacion Cubanas. Cuba: Universidad de Granada. Recuperado el 3 de enero de 2016, de http://digibug.ugr.es/bitstream/10481/34252/1/23997461.pdf

Rodriguez, E. V. (2012). Finanzas corporativas en los nuevos empresarios. España: Nube.

Saaty, T. (2002). Investigacion de operaciones y la toma de decisiones. Iraq: ANP.

Simon, H. A. (1999). Administrative Behavior: A Study of Decision-making Processes in Administrative Organization. Estados Unidos: Academic Press.

Superintendencia de Banca del Perú. (2012). Determinacion de los Beneficios de la Educacion Financiera. Perú: Aguilar.

Torres Higuerra, R. (2009). La importancia de la educacion financiera en las inversiones y el credito . Mexico: FCA.

Valdiviezo. (2007). La Toma de Decisiones. Mexico: Aguilar.

Recibido: 20/10/2016

Aceptado para la publicación

$7 / 11 / 2016$ 